Recepción: 18/02/2019

Aceptación: 22/03/2019

Ciencias de la educación

Publicación: 05/05/2019

Artículo de revisión

\title{
La tutoría entre iguales: Modalidad para promover el aprendizaje cooperativo a nivel superior
}

Peer tutoring: Modality to promote cooperative learning at a higher level

\section{Tutoria de pares: Modalidade para promover a aprendizagem cooperativa em um nível superior}

\author{
Mayra M. Palma-Villavicencio ${ }^{\mathrm{I}}$ \\ mpalma@utm.edu.ec \\ Liliana del R. Loor-Salmon II \\ 1loor@utm.edu.ec
Letty J. Saltos-Rodríguez III 1saltos@utm.edu.ec \\ Oscar E. Bolívar-Chávez ${ }^{\mathrm{IV}}$ \\ obolivar@utm.edu.ec
}

Correspondencia:mpalma@utm.edu.ec

I. Profesora de Segunda Enseñanza, Docente del Instituto de Lenguas de la Universidad Técnica de Manabí, Portoviejo, Ecuador.

II. Magíster en Docencia e Investigación Educativa, Doctora en Educación, Licenciada en Ciencias de la Educación Especialidad Inglés, Profesora de Segunda Enseñanza Idiomas y Lingüística Especialidad Inglés, Docente del Instituto de Lenguas de la Universidad Técnica de Manabí, Portoviejo, Ecuador.

III. Magíster en Enseñanza del Idioma Inglés, Licenciada en Ciencias de la Educación Mención Inglés Francés, Docente del Instituto de Lenguas de la Universidad Técnica de Manabí, Portoviejo, Ecuador.

IV. Ph.D. en Educación. Máster en Nuevas Tecnologías Aplicadas a la Educación, Licenciado en Ciencias de la Educación Especialidad Instrumentista Pedagogo en Saxofón. CETAC, Docente Universidad Técnica de Manabí, Portoviejo, Ecuador. 


\section{Resumen}

La tutoría entre iguales es una modalidad pedagógica en la que estudiantes, generalmente de semestres más avanzados, acompañan a sus pares en la orientación y el refuerzo de los procesos de aprendizaje dentro de un área disciplinar, el cual permite la promoción del aprendizaje cooperativo en el nivel de educación superior. Ante este planteamiento, el objetivo de este artículo se centró en evaluar la tutoría entre iguales como una modalidad para promover el aprendizaje cooperativo a nivel superior. El estudio siguió una metodología de tipo descriptiva con un diseño no experimental y de campo, puesto que fue necesario estudiar los hechos donde se originan para observarlo, describirlos y aplicar los instrumentos necesarios para la obtención de los resultados. El diseño de esta investigación se consideró no experimental y de campo. La población la constituyeron 14 estudiantes que ejercían el rol de tutores entre iguales. Se observaron y recolectaron los datos a través de la técnica de la observación participativa, para ello se utilizó como instrumentos una entrevista estructura y un cuestionario con una escala tipo Likert. Entre sus resultados, se destaca el hecho en la cual el porcentaje más alto con el $82 \%$ de los investigados, indican que la falta de profesores que les apoyen en su labor no les permite ayudar con más efectividad a sus pares estudiantes. Se pudo concluir que la tutoría entre iguales, como una modalidad para promover el aprendizaje cooperativo a nivel superior, no cumple su rol en los espacios universitarios investigados de Portoviejo.

Palabras clave: Tutoría entre iguales; aprendizaje cooperativo; docente y estudiantes.

\section{Abstract}

Peer tutoring is a pedagogical modality in which students, usually from more advanced semesters, accompany their peers in the orientation and reinforcement of learning processes within a disciplinary area, which allows the promotion cooperative learning at the higher education level. In view of this approach, the objective of this article focused on evaluating peer mentoring as a modality for promoting cooperative learning at the higher level. The study followed a descriptive methodology with a non-experimental and field design, since it was 
necessary to study the facts where they originate to observe it, describe them and apply the necessary instruments to obtain the results. The design of this research was considered nonexperimental and fielded. The population was made up of 14 students who played the role of peer tutors. The data was observed and collected through the participatory observation technique, for this purpose a structure interview and a questionnaire with a Likert scale were used as instruments. Among their results is the fact that the highest percentage with $82 \%$ of those researched, indicate that the lack of teachers who support them in their work does not allow them to help their student peers more effectively. It could be concluded that peer mentoring, as a modality for promoting cooperative learning at the higher level, does not fulfil its role in the researched university spaces of Portoviejo.

Keywords: Peer tutoring; cooperative learning; teachers and students.

\section{Resumo}

A tutoria por pares é uma modalidade pedagógica em que os alunos, geralmente de semestres mais avançados, acompanham seus pares na orientação e reforço dos processos de aprendizagem dentro de uma área disciplinar, o que permite a promoção da aprendizagem cooperativa na nível de ensino superior. Dada esta abordagem, o objetivo deste artigo focou-se em avaliar a tutoria entre pares como uma modalidade para promover a aprendizagem cooperativa em um nível superior. O estudo seguiu uma metodologia descritiva com delineamento não experimental e de campo, pois foi necessário estudar os fatos de onde se originaram para observá-lo, descrevê-los e aplicar os instrumentos necessários para a obtenção dos resultados. O desenho desta pesquisa foi considerado não experimental e de campo. A população foi composta por 14 estudantes que exerceram o papel de tutores pares. Os dados foram observados e coletados por meio da técnica 
de observação participativa, para isso foram utilizados como instrumento uma entrevista estruturada e um questionário com escala Likert. Entre seus resultados, vale destacar o fato de que o maior percentual com $82 \%$ dos pesquisados indica que a falta de professores que os apoiam em seu trabalho não lhes permite ajudar seus pares de maneira mais efetiva. Concluiu-se que a tutoria por pares, como modalidade de promoção da aprendizagem cooperativa em nível superior, não cumpre seu papel nos espaços universitários pesquisados de Portoviejo.

Palavras-chave: Tutoria de pares; aprendizagem cooperativa; Professor e alunos.

\section{Introducción}

La tutoría entre iguales es una modalidad estratégica de carácter pedagógica en la que estudiantes de semestres superiores, acompañan a sus pares para reforzar y dar orientación acerca de los contenidos previstos o ya alcanzados en materia académica, esto ocurre en pequeños grupos. Por su parte, el profesorado apoya y valora la labor de tutoría con el material que requieran dejándoles la autonomía suficiente como para que lo lleven a cabo sin un guía docente.

Topping (2000), explica que la tutoría entre iguales es ampliamente utilizada en muchos países, tanto en la educación formal como en la informal, así como en todos los niveles educativos y áreas curriculares. Es recomendada por expertos en educación, por ejemplo, la UNESCO, como una de las prácticas instructivas más efectivas para la educación de calidad.

Asimismo, Tokuhama y col (2009) agregan que los estudiantes aprenden un 5\% de lo que escuchan, un $10 \%$ de lo que leen, un $20 \%$ de lo que ven, un $30 \%$ en demostraciones, un $50 \%$ en discusión de grupos, pero al practicar haciéndolo aprenden hasta el 75\% y si enseñan a otros hasta un 90\%, de allí que siempre se dice que aprende más el que enseña que el que aprende. 
Esta premisa, permite pensar que existe un aprendizaje cooperativo como herramienta pedagógica, que fortalece los procesos de aprendizaje. Sin embargo, en las universidades de Portoviejo, es posible observar alumnos que requieren de este tipo de apoyo sin las condiciones para ello, a pesar de encontrar tutores en sus pares. Esta posición, permitió evaluar la tutoría entre iguales como una modalidad para promover el aprendizaje cooperativo a nivel superior.

\section{Desarrollo}

Los métodos de aprendizaje cooperativo organizan los pequeños grupos con el objetivo de establecer los vínculos y requisitos necesarios para la cooperación. Pero a pesar de tener eso en común, cada uno representa una manera diferente de gestionar las actividades de enseñanza aprendizaje, lo cual hace que unos sean más adecuados que otros para desarrollar determinados aprendizajes en las diferentes áreas curriculares. No hay método mejor ni más adecuado que los otros, se trata de utilizar en cada momento, aquel que se adapte más a nuestras necesidades en función del grupo de alumnos y la actividad a desarrollar de manera que se potencien los factores que faciliten la cooperación y el aprendizaje. Sin embargo, cuando se trata de trabajar en parejas podríamos señalar la "tutoría entre iguales" los alumnos tutores de cada pareja enseñan y/o ayudan a su compañero en la realización de ejercicios o actividades propuestas en una determinada área de conocimiento.

\section{Tutoría entre iguales}

El modelo de las tutorías entre iguales, evaluado en la universidad se fundamentó en los siguientes pilares, presentado por Morales (2009).

La flexibilidad: permite dar respuesta a la adaptación a las necesidades diversas de un estudiante diverso. El perfil de los estudiantes requiere de un sistema que pueda adaptarse de su realidad personal, profesional y familiar. Dicha flexibilidad debe verse reflejado en el modelo de evaluación, en el Plan de Trabajo de las asignaturas, la del acceso a las fuentes de información, es decir, debe estar pensado para satisfacer a unos estudiantes que necesitan de un sistema que se adapte a sus necesidades y no al revés. 
La cooperación: refiere al acompañamiento docente que requieren los estudiantes para no estar solos en su proceso de aprendizaje. Todo ello, realizando trabajos en equipo, o con los consultores, o con personas diversas de la comunidad universitaria en la creación de grupos de interés.

La personalización: facilita el trato individual de las necesidades formativas de cada estudiante. Refiere una manifestación de trato personalizado en el proceso de aprendizaje, en el que tanto los materiales multimedia de aprendizaje como los consultores tratan de forma personal al estudiante en sus necesidades formativas.

La interactividad: La interacción es múltiple ya que no sólo abarca la acción docente, sino que además se establece entre estudiantes y entre estos con la propia universidad.

\section{Ámbitos del plan de Tutoría entre Iguales}

Para Álvarez (2009), la tutoría entre iguales atiende a cuatro ámbitos de intervención diferentes:

Ámbito Académico: implica el protagonismo del alumnado en su propio proceso de formación. Se pretende asesorar al alumnado de nuevo ingreso sobre las opciones de formación académica, el sentido que tienen las materias en el plan de estudios, las exigencias de cada materia y cómo responder a ellas; todo esto desde la visión y experiencia del estudiante "veterano".

Ámbito de Gestión: con el que se pretende informar sobre los diferentes órganos de gestión de la vida universitaria y fomentar la participación del alumnado en la misma.

Ámbito Administrativo: en el que se facilitará el conocimiento y acceso a la información administrativa que se genere, así como los medios, canales y recursos que dispondrán para su transmisión.

Ámbito de Servicios: en el que se dará a conocer y motivará al alumnado hacia el uso de los diferentes servicios que pone a su disposición la Universidad.

\section{Aprendizaje Cooperativo}

El aprendizaje cooperativo siempre ha formado parte de las interacciones humanas, no obstante, es en los años 80 cuando el aprendizaje cooperativo golpeó fuerte como método de enseñanza a 
través de los primeros metaestudios sobre los efectos en las escuelas de los Estados Unidos. Mayer (1929), citado por Morales (2010), mostró que la cooperación es más eficaz que la competición y Johnson y Johnson $(1975,1987$ y 1990) que la estructura cooperativa, con relación a la competitiva e individualista, produce un mayor rendimiento en el aprendizaje de los alumnos, desarrolla unas actitudes más positivas hacia la escuela y hacia los profesores y compañeros, se consiguen niveles más elevados de autoestima, y favorece más el desarrollo de la motivación intrínseca.

\section{Ventajas del Aprendizaje Cooperativo}

- Se incrementa la motivación, las interacciones, los alumnos colaboran y aprenden unos de otros, equilibrándose el ritmo de trabajo en un ambiente general de autosuperación.

- Los estudiantes están motivados a dar lo mejor de sí para contribuir a los éxitos de los demás o, en su caso, del equipo.

- Fomenta el autoaprendizaje, ya que los alumnos seleccionan la información y crean sus propios contenidos.

- Ayuda a mejorar la empatía y el asertividad.

- Produce entornos educativos que favorecen el interés y la implicación.

\section{Desventajas del Aprendizaje Cooperativo}

- Las etapas previas a la fijación de objetivos y la elaboración de un esquema de trabajo claro pueden alargarse con discusiones y desacuerdos.

- Buena parte del trabajo se realiza en ausencia del docente facilitador, en la que algunas dudas pueden tardar en aclararse, empeora el ambiente de clases.

- Los grupos presentan desequilibrios internos que es complicado compensar. Se producen subgrupos y el efecto líder.

- Es fácil que surja la desmotivación por la sensación de pérdida de tiempo.

- Los grupos se encasillan en roles y en sistemas de trabajo, incluso cuando son ineficientes.

\section{Metodología}

El estudio siguió una metodología de tipo descriptiva, en el mismo se consideró la tutoría entre iguales como una modalidad para promover el trabajo cooperativo en universidades del Cantón 
de Portoviejo en la provincia de Manabí. Las investigaciones descriptivas según Silva (2010), buscan caracterizar el objeto de estudio en una situación concreta, describiendo las cosas tal cual se dan en el presente. El diseño de esta investigación se consideró no experimental y de campo, puesto que fue necesario estudiar los hechos donde se originan para observarlo, describirlos y aplicar los instrumentos necesarios para la obtención de los resultados. Se observaron y recolectaron los datos a través de la técnica de la observación participativa, para ello se utilizó como instrumentos una entrevista estructura y un cuestionario con una escala tipo Likert, el cual se aplicó a 14 estudiantes que cumplen el rol de tutores.

\section{Resultados}

\section{Entre los resultados se pueden mencionar:}

\section{a.- En relación a la entrevista estructura se pudo constatar:}

- El aprendizaje cooperativo, según el $72 \%$ de los estudiantes que cumplen el rol de tutores funciona bien cuando logran preparar adecuadamente el entorno, afirmando que cuentan con espacios no adecuados, para que los estudiantes a atender puedan alejarse y acercarse.

- El $82 \%$ de los investigados, indican que la falta de profesores que les apoyen en su labor no les permite ayudar con más efectividad a sus pares estudiantes, pues en algunas oportunidades requieren saber según los casos de contenido programático a impartir cual podría ser el mejor y más práctico método de aplicación. 
- Se pudo constatar entre los 14 estudiantes investigados que el 50\% de ellos, consideran necesario que posterior a su labor, deben ser evaluados por sus pares con la finalidad de analizar cómo ha sido el trabajo, que roles se han asumido y qué ineficiencias se han observado, todo ello para mejorar o cubrir las que se hayan dado durante la tutoría.

\section{b.-En cuanto al instrumento que midió la tutoría entre iguales, lo siguiente:}

Tabla 1 Análisis descriptivo frecuencial de la variable Tutoría entre iguales

\begin{tabular}{|c|c|c|c|c|c|c|c|c|c|c|}
\hline \multirow[t]{2}{*}{$\begin{array}{l}\text { NIVEL } \\
\end{array}$} & \multicolumn{2}{|c|}{ Siempre } & \multicolumn{2}{|c|}{$\begin{array}{c}\text { Casi } \\
\text { Siempre }\end{array}$} & \multicolumn{2}{|c|}{$\begin{array}{l}\text { Algunas } \\
\text { veces }\end{array}$} & \multicolumn{2}{|c|}{ Casi nunca } & \multicolumn{2}{|c|}{ Nunca } \\
\hline & Fr & $\%$ & Fr & $\%$ & Fr & $\%$ & Fr & $\%$ & Fr & $\%$ \\
\hline Flexibilidad & 3 & 21 & 6 & 43 & 5 & 36 & 0 & 0 & 0 & 0 \\
\hline Cooperación & 0 & 0 & 1 & 7 & 5 & 36 & 7 & 50 & 1 & 7 \\
\hline Personalización & 0 & 0 & 4 & 28 & 8 & 58 & 2 & 14 & 0 & 0 \\
\hline Interactividad & 0 & 0 & 0 & 0 & 2 & 14 & 9 & 65 & 3 & 21 \\
\hline
\end{tabular}

Fuente: Elaboración propia. 


\section{Grafico 1. Análisis descriptivo frecuencial de la variable Tutoría entre Iguales}

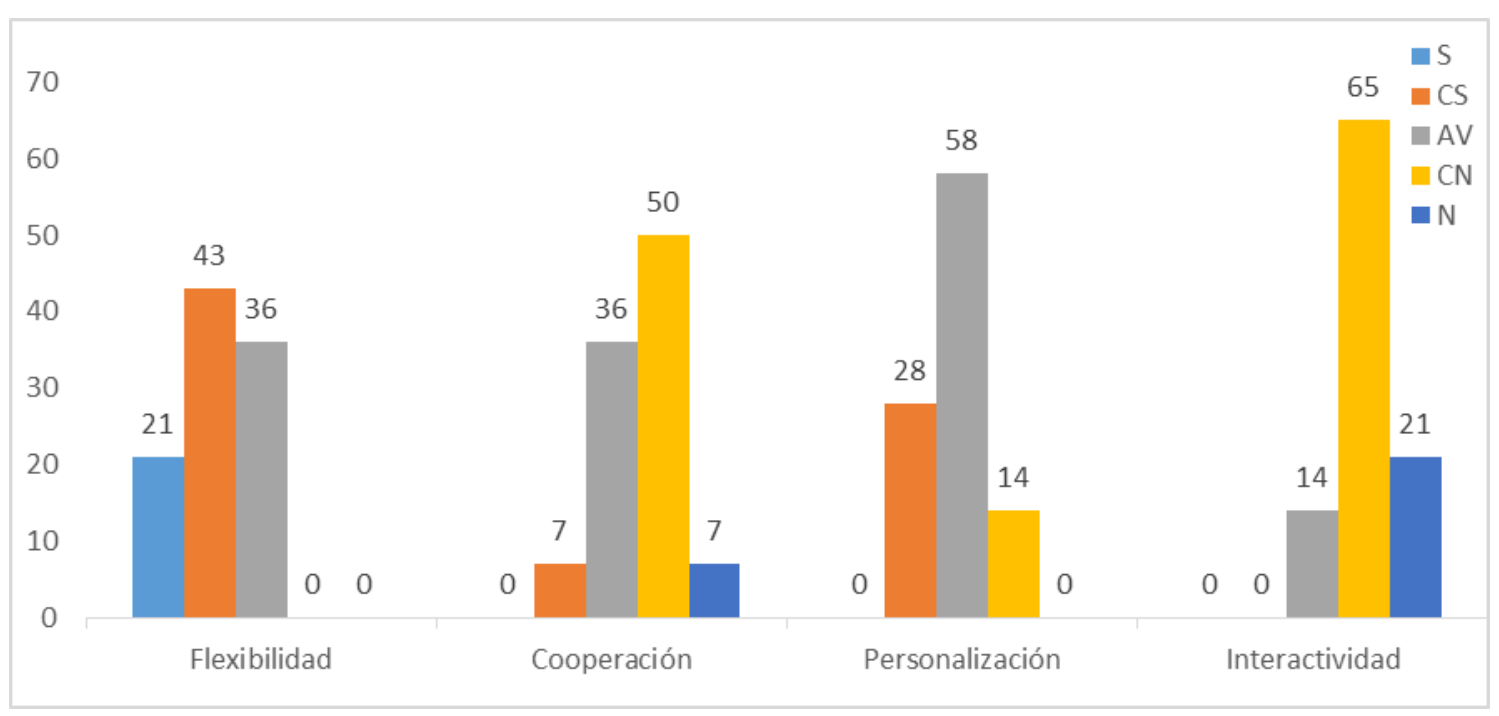

Fuente: Elaboración propia.

En la tabla y grafico 1, se presentan los resultados del análisis descriptivo frecuencial de la variable tutoría entre iguales; en la misma se observa que el $43 \%$ de los investigados indicaron que algunas veces se cumple, seguido del $36 \%$ que señalaron la alternativa algunas veces. En cuanto al indicador Cooperación, se indica que el 50\% considero que casi nunca se da el acompañamiento docente que requieren los estudiantes tutores. Para el indicador Personalización se pudo constatar que el 58\% de los investigados consideran que casi nunca logran personalizar el trato de sus pares. Finalmente, en cuanto al indicador interactividad el $65 \%$ afirmo que casi nunca la logran con sus pares ni profesores. 


\section{Conclusiones}

La tutoría entre iguales como una modalidad para promover el aprendizaje cooperativo a nivel superior, no cumple su rol en los espacios universitarios investigados de Portoviejo. Este hecho encuentra su evidencia, al no garantizar la preparación de sus espacios académicos para desempeñarse como tutores de sus pares y el poco interés que demuestran los docentes de las distintas cátedras para apoyar este proceso, por lo que ellos solicitan ser evaluados y de forma auto correctivas mejorar en este rol.

Casi siempre la flexibilidad, en la tutoría entre iguales, logra su adaptación a las necesidades diversas de un estudiante diverso, en la que se requiere saber el perfil de los estudiantes para poder adaptarse a la realidad personal, profesional y familiar de cada uno de ellos y de esta manera facilitar el proceso tutorial

Casi nunca, en la tutoría entre iguales, se cumple con el pilar de la cooperación, al carecer del acompañamiento docente que requieren los estudiantes para no estar solos en su proceso de aprendizaje.

Solo algunas veces es posible dar cumplimiento al pilar de la personalización durante la tutoría entre iguales, que permita facilitar el trato individual de las necesidades formativas de cada estudiante y que debe ser de su conocimiento para el trato personalizado durante el proceso de aprendizaje.

Casi nunca se cumple con el pilar de la interactividad durante la tutoría entre iguales, La interacción es múltiple ya que no sólo abarca la acción docente, sino que además se establece entre estudiantes y entre estos con la propia universidad. 


\section{Referencias Bibliográficas}

Álvarez, P.R. et al. "La tutoría entre iguales como estrategia para la adquisición de competencias genéricas en la enseñanza superior". V Congreso Internacional de Formación para el Trabajo. Publicación CD-ROOM, Granada 2009

Arco, J. L.; Fernández, F.D. "Eficacia de un programa de tutoría entre iguales para la mejora de los hábitos de estudio del alumnado universitario". Revista de Psicodidáctica. 2011, vol. 16, núm. 1, p. 163-180

Fernández, F. D., Arco, J. L., Ortega, S. y Heilborn, V. A. (2011). Prevención del fracaso académico universitario mediante tutoría entre iguales. Revista Latinoamericana de Psicología, vol.43 (1), 59-71.

Morales Hernández (2009). Entrevista personal. Directora Administrativa del Instituto de Educación a Distancia. Consulta por Correo Electrónico realizada el 15-Mar-2009.

Silva, Jesús (2010). Metodología de la Investigación: Elementos Básicos. Caracas, Venezuela. Editorial Litho-Tip, C.A. Págs. 20, 21.

Tokuhama -Espinoza, Tracey, Mosquera, Jean-Michael y Virginia Sanguinetti. (2009) ¿Cómo aprenden los adultos? Disponible en: http://www.educacionparatodos.com/pdf/2.pdf 
Mayra M. Palma Villavicencio, Liliana del R. Loor Salmon, Letty J. Saltos Rodríguez, Oscar E. Bolívar Chávez

Topping, K.(2000). Family and Volunteers. Geneva: International Bureau of Education, UNESCO. Disponible en: http://www.ibe.unesco.org/publications/practices.htm 